\title{
Characteristic Size of Molecular Dynamics in Polymers Probed by Dielectric Probes of Variable Length.
}

\author{
Otto van den Berg, Michael Wübbenhorst*, Stephen J. Picken and Wolter F. Jager
}

Delft University of Technology, Faculty of Applied Sciences, Department of Polymer Materials and Engineering, Julianalaan 136, 2628BL Delft, The Netherlands. The Dutch Polymer Institute

\section{Abstract}

Recently we have introduced a rigid-rod type chromophore, E-4,4'- $N, N$-dibutylamino-nitro-stilbene (DBANS), that acts as a dielectric and fluorescent probe, and enables the study of molecular dynamics in apolar polymers by dielectric relaxation spectroscopy (DRS). This work focuses on the effect of the probe size and concentration on the specific probe response. For this purpose, a series of probe molecules with core lengths $(l)$ between 0.6 and $2 \mathrm{~nm}$ was used to 'dope' polystyrene at concentrations from 0.1 to $1.0 \mathrm{wt} \%$. For all probes, a selective amplification of the intrinsic $\alpha$-relaxation was found that was linear within the whole probe concentration range. Moreover, two clear size effects on the probe dynamics were revealed: At high temperatures, the probe relaxation time obeys $\tau \propto l^{3}$, which confirmed rotational diffusion as the linking mechanism between the polymeric segmental dynamics and dielectric probe response.

The shortest probe revealed an additional Arrhenius-type $\left(\beta^{*}\right)$ relaxation that was attributed to large angular probe fluctuations involving discrete jumps. Its exclusive occurrence indicates a critical lower probe size $\left(l^{*} \sim 0.6 \mathrm{~nm}\right)$ below which the dielectric probe acts also as a "free-volume probe" as indicated by the pronounced anormality in the $\tau_{\beta *}(T)$ dependence.

Finally, an attempt was made to relate probe concentration effects on the glass transition temperature (plasticizing effect) to the intrinsic length scale of cooperative dynamics $\xi$. Based on a simple model for local plasticization we have determined a lower bound for $\xi$ in the order of $5 \mathrm{~nm}$.

\section{Keywords}

Dielectric Relaxation, Glass Transition, Polymer Dynamics, Fluorescent probe, Cooperativity

*Corresponding author 


\section{Introduction}

In the past decade broadband dielectric spectroscopy (DRS) has been widely used for the study of molecular dynamics in many liquids and polymers because of the easy accessibility of more than 15 decades in frequency [1-4]. By virtue of probing orientation fluctuations of molecular dipoles, DRS allows to study molecular motions on various length scales ranging from individual bond rotations, the cooperative glass transition up to relaxation processes involving the contour of the entire polymer coil (normal process). Since DRS spans a wide frequency range and can easily be performed at different temperatures, this technique yields a wealth of information on molecular dynamics in the solid and liquid state.

Typical examples of polymers studied by DRS include traditional amorphous (PMMA, PC) and semi-crystalline polymers (PET, PEN, PEO) [2, 5, 6], complex, (multi-phase) liquid-crystalline polymers [7, 8] as well as nano-composites or supra-molecular polymers.[9] A prerequisite for the application of dielectric relaxation spectroscopy is the presence of molecular dipoles, which provide the necessary link between the molecular motions and an external electrical (probing) field. Though most polymer systems contain at least weakly polar groups, either in the main-chain or the side-chain, there is a substantial class of non-polar polymers like polyolefins, which do not contain dipolar moieties. To overcome this problem, various methods that aim to introduce permanent dipoles have been proposed $[10,11]$.

In a recent paper [12] we have demonstrated that $N, N$-dibutylamino- $E$-nitrostilbene (DBANS, cf. Figs. 1 and 2), a polar rigid rod-like chromophore, acts as a suitable "dielectric probe" for the study of cooperative dynamics, when dissolved in an apolar polymer like PE, PP or PS. Due to the lack of internal degrees of freedom of DBANS that could give rise to intrinsic probe relaxations, no dielectric activity of the probe was found in the glassy state. Above $T_{\mathrm{g}}$, the cooperative segmental motions, i.e. the dynamic glass transition, cause large scale fluctuations of the polar axis of the rigid probe molecules, while other, local processes and the dynamics involving the crystalline phase do not affect the dielectric probe response. In polystyrene, the dielectric probe "amplifies" the intrinsic relaxation behavior of the matrix polymer by specifically enhancing the dielectric glass transition process. The increase in dielectric strength was shown to follow linearly the probe concentration up to about $1.0 \%$. The relaxation time of the probe coincided with the mean relaxation time of undoped polystyrene within one order of magnitude in frequency.

\section{Insert figure 1.}

For an optimal performance, a dielectric probe molecule has to satisfy a number of requirements: i) First, the probe molecule must be rigid to eliminate dielectric relaxations associated to 
intramolecular motions, ii) it should have a large permanent dipole moment and iii) it must possess a sufficient solubility in an apolar environment.

The aim of our current research is to explore the dielectric probe approach as a generic technique to sensitize non-polar polymers for dielectric spectroscopy investigations. In a previous paper [12] we have reported the use of DBANS $(\boldsymbol{B})$ as a dielectric probe in LDPE, $i$-PP and PS. Here we focus on the effect of the probe length and concentration on the dielectric response in atactic polystyrene (PS). The weak polarity of the polymer, due to the small dipole moment of the phenyl ethylene moieties, allows for a direct comparison between the intrinsic dielectric relaxation processes of the pristine polymer with those of the doped polymer. Three types of probe molecules, having different lengths of the rigid core $l_{\mathrm{c}}$ of $0.64,1.3$ and $2.0 \mathrm{~nm}$ (cf. Fig. 2), were studied in combination with PS as the polymer matrix. These molecules all possess relative large dipole moments [13], while the aliphatic tails provide these molecules with a good solubility in non-polar media.

\section{Insert figure 2}

\section{Experimental}

\subsection{Sample preparation}

Polystyrene was received from Shell (PS, N7000) and was purified by a triple precipitation from dichloromethane/methanol. Mixtures of polystyrene with probe were prepared by dissolving in spectroscopic grade dichloromethane and solution casting on a glass plate. The plates were left to dry for 24 hours at room temperature, 48 hours at $50^{\circ} \mathrm{C}$ and $20 \mathrm{mmHg}$. The sheets were cut with a razor blade and a drop of water was added to release the sheets from the glass. Squared pieces of $1 \mathrm{~cm}^{2}$ were cut and pressed together with $100 \mu \mathrm{m}$ glass fiber spacers between circular brass electrodes $(\varnothing=2 \mathrm{~cm})$ resulting in samples with a well-defined geometry for DRS experiments.

\subsection{Differential scanning calorimetry}

DSC heat flow curves of all samples were recorded with a Perkin Elmer DSC-7. Indium was used for temperature calibration. All samples were annealed for 5 minutes at $220^{\circ} \mathrm{C}$ and subsequently cooled at a rate of $10 \mathrm{~K} / \mathrm{min}$. Glass transition temperatures were determined from the $C_{\mathrm{p}}$ inflection point via differentiation.

\subsection{Dielectric relaxation spectroscopy}


Dielectric experiments were performed using a high precision dielectric analyzer (ALPHA analyzer, Novocontrol Technology) in combination with a Novocontrol Quatro temperature system providing control of the sample temperature with an accuracy better than $50 \mathrm{mK}$. All dielectric measurements were performed in the frequency range from $10^{-1}$ to $10^{7} \mathrm{~Hz}$. Temperature dependent experiments were realized by consecutive isothermal frequency sweeps in the temperature range from $+200^{\circ} \mathrm{C}$ to $-120^{\circ} \mathrm{C}$ in steps of $-5 \mathrm{~K}$, which resulted in an effective cooling rate of about $0.5 \mathrm{~K} / \mathrm{min}$. More experimental details can be found in [5] and references therein. In order to determine the relaxation time $\tau(T)$ from the dielectric loss curves we have fitted the frequency spectra $\varepsilon^{\prime \prime}(\omega)$ by the empirical HavriliakNegami [14] relaxation function (eq. 1) using the Levenberg-Marquardt [15, 16] algorithm:

$$
\varepsilon^{\prime \prime}=-\operatorname{Im}\left\{\frac{\Delta \varepsilon}{\left(1+(i \omega \tau)^{a}\right)^{b}}\right\}+\frac{\sigma}{\varepsilon_{0} \omega}
$$

Here $\Delta \varepsilon$ and $\tau$ correspond to the relaxation strength and the mean relaxation time of the relaxation process. The two shape parameters $a$ and $b$, which determine the logarithmic slope of the low frequency loss tail $a$ and the high frequency loss tail $-a \cdot b$, are determined by the underlying distribution in relaxation times. The second term in eq. 1 accounts for ohmic conduction.

\section{Results}

\subsection{Differential Scanning Calorimetry}

Differential Scanning Calorimetry (DSC) is a generally accepted technique for measuring thermal transitions like glass transitions or crystallisation/melting phenomena. In this study, DSC was used to investigate the effect of probe addition on the glass transition temperature. DSC measurements on the PS samples showed a clear step in $c_{\mathrm{p}}$ at about $100^{\circ} \mathrm{C}$, from which the glass transition temperature is derived. Upon addition of probe $\boldsymbol{A}$ and $\boldsymbol{B}, T_{\mathrm{g}}$ values shift gradually to lower temperatures as the probe contents increases. Typical $T_{\mathrm{g}}$ depressions by $6-7^{\circ} \mathrm{C}$ for samples containing $1 \%$ of probe $\boldsymbol{A}$ or $\boldsymbol{B}$ are indicative for a slight but significant plasticizing effect of these probe molecules. Addition of probe $\boldsymbol{C}$, however, did not reveal any significant effect on the glass transition temperature. 
Table 1. DSC glass transition temperatures determined from the half $c_{p}$ values.

\begin{tabular}{lcc}
\hline & $C_{\text {Probe }}[17]$ & $T_{\mathrm{g}}{ }^{a}[18]$ \\
\hline PS & 0 & 99.5 \\
\hline PS and $\boldsymbol{A}$ & 0.1 & 98.1 \\
& 0.25 & 95.5 \\
& 0.5 & 94.8 \\
& 0.75 & 94.0 \\
& 1 & 91.9 \\
\hline PS and $\boldsymbol{B}$ & 0.1 & 98.0 \\
& 0.5 & 96.1 \\
& 1 & 93.6 \\
\hline PS and $\boldsymbol{C}$ & 0.1 & 98.9 \\
& 0.25 & 98.9 \\
& 0.5 & 100.3 \\
& 1 & 98.4
\end{tabular}

a) The DSC glass transition temperatures show a typical experimental error of $0.5^{\circ} \mathrm{C}$

\subsection{Dielectric relaxation spectroscopy}

\section{Loss spectra of PS containing probes $B$ and $C$ :}

In Figure 3 the isochronal representations of the dielectric loss, i.e. $\varepsilon^{\prime \prime}(T)$ at a fixed frequency $(f=13$ $\mathrm{kHz}$ ), for samples with various concentrations of the dielectric probes $\boldsymbol{B}$ and $\boldsymbol{C}$ are shown. The graphs reveal a number of characteristic features. At about $-80^{\circ} \mathrm{C}$ the dielectric $\beta$-process of PS is visible as a broad peak. This process that is usually attributed to crankshaft-type motions in the backbone involving the polar phenyl moiety [1, 19], is obviously not affected by the presence of either $\boldsymbol{B}$ or $\boldsymbol{C}$. At about $140^{\circ} \mathrm{C}$, a second loss peak corresponding to the dielectric $\alpha$-process of PS, the dielectric manifestation of the dynamic glass transition, shows up. This process, which is also clearly discernible for undoped PS, gains further intensity upon addition of probe molecules $\boldsymbol{B}$ or $\boldsymbol{C}$. A closer look on figure 3 shows that this peak enhancement is accompanied by systematic shifts in the peak maximum temperature, which depend on the probe type and the probe concentration. For probe $\boldsymbol{B}$, a gradual down-shift of the $\alpha$-peak maximum can be recognized, which is in line with the weak plasticising effect concluded from the DSC data (cf. Table 1).

Apart from the weak concentration dependence of the peak maximum temperature (plasticisation), one easily sees that the maxima for the loss peak are found at significantly higher temperatures for the longest probe $\boldsymbol{C}$ as compared to $\boldsymbol{B}$. Such shift towards higher temperatures seems to be plausible, when we assume a general relationship between the relaxation time and the length scale of molecular 
motions. Hence, in a homogeneous mobility scenario, a longer probe $(\boldsymbol{C})$ will "see" slower molecular reorganisations than the shorter probe $\boldsymbol{B}$.

\section{Insert figure 3.}

\section{Loss spectra of PS doped with probe A:}

Figure 4 depicts the analogous loss curve $\varepsilon^{\prime \prime}(T, f=13 \mathrm{kHz})$ for PS doped with the dielectric probe $\boldsymbol{A}$. At about $40^{\circ} \mathrm{C}$ a new, broad relaxation peak emerges, the intensity of which clearly depends on the probe concentration. Due to its location between the $\beta$-process, which has a peak maximum around $80^{\circ} \mathrm{C}$, and the $\alpha$-process, we have labeled this new relaxation process $\beta^{*}$. At high temperatures, this process interferes partially with the dielectric $\alpha$-process of PS. Although Figure 4 itself is not entirely convincing at this point, the $\alpha$-relaxation is amplified by the addition of probe $\boldsymbol{A}$ as well, however less efficient than upon addition of $\boldsymbol{B}$ or $\boldsymbol{C}$.

\section{Insert figure 4.}

\section{Activation energies of probe related dielectric processes $(A, B$ and $C)$ :}

In order to identify the various relaxation processes according to their thermal activation, we have determined the relaxation times at the loss peak maxima from the $\mathrm{HN}$-fit using equation 1 . All $\tau(T)$ data corresponding to the $\alpha$ and $\beta^{*}$-relaxation are presented in an Arrhenius diagram (Figure 5). While the $\alpha$-process shows the typical curvature according to the Vogel-Fulcher-Tammann (VFT) law (Eq.2) [20-22], being indicative for the dynamic glass transition, two temperature regions can be identified, in which the $\beta^{*}$-relaxation obeys the Arrhenius law (Eq. 3).

$$
\begin{aligned}
& \tau=\tau_{\infty} \exp \left(\frac{E_{v}}{R\left(T-T_{V}\right)}\right) \\
& \tau=\tau_{\infty} \exp \left(\frac{E_{a}}{R T}\right)
\end{aligned}
$$

A striking feature of the $\beta^{*}$ process is the kink at about $80^{\circ} \mathrm{C}$, which separates a low temperature Arrhenius part $\left(E_{\mathrm{a}}=50-65 \mathrm{~kJ} / \mathrm{mol}, \tau_{\infty}=10^{-15} \mathrm{~s}\right)$ from a high temperature branch with an activation 
energy of approximately $135 \mathrm{~kJ} / \mathrm{mol}\left(\tau_{\infty}=10^{-25} \mathrm{~s}\right)$. This large increase of the activation energy of the $\beta^{*}$-process around $T_{\mathrm{g}}$ was observed for all samples.

\section{Insert figure 5.}

Table 2. Dielectric glass transition temperatures derived from VFT data.

\begin{tabular}{lccc}
\hline & cProbe $\%$ & $\begin{array}{c}\text { "dielectric" } \\
(\tau=100 \mathrm{~s})[18]\end{array}$ & $\mathrm{T}_{\mathrm{g}}$ \\
\hline PS & 0.1 & $96.6 \pm 1$ \\
\hline PS and $\boldsymbol{A}$ & 0.25 & $98.4 \pm 1$ \\
& 0.5 & $97.5 \pm 1$ \\
& 1 & $96.9 \pm 1$ \\
\hline PS and $\boldsymbol{B}$ & 0.1 & $94.3 \pm 1.5$ \\
& 0.5 & $95.0 \pm 1$ \\
& 1 & $93.4 \pm 1$ \\
\hline PS and $\boldsymbol{C}$ & 0.1 & $90.1 \pm 2$ \\
& 0.25 & -- \\
& 0.5 & -- \\
& 1 & $98.7 \pm 1$ \\
\end{tabular}

From the VFT curves displayed in Figure 5 one can evaluate an operationally defined "dielectric" glass transition temperature being defined as the temperature where the relaxation time $\tau$ becomes 100 s. The results are listed in Table 2 showing a fair agreement between the dielectric $T_{\mathrm{g}}$-values derived from DRS and DSC, e.g. $T_{\mathrm{g}}=96.6^{\circ} \mathrm{C}$ and $99.5^{\circ} \mathrm{C}$ for undoped PS. Plasticizing effects, resulting in $T_{\mathrm{g}}$ depressions by $2-6^{\circ} \mathrm{C}$ upon addition of $1 \%$ probe, are observed for all probes.

\section{Relaxation strength}

The relation between the probe concentration and the dielectric relaxation strength of the $\alpha$ relaxation, $\Delta \varepsilon_{\alpha}$, is shown Figure $6 \mathrm{a}$. Although the effect of probe addition depends on the specific probe type, the $\Delta \varepsilon_{\alpha}(\mathrm{c})$ dependence reveals a clear linearity for probes $\boldsymbol{A}, \boldsymbol{B}$ and $\boldsymbol{C}$. The remaining, finite $\Delta \varepsilon_{\alpha}$ value at zero probe concentration is due to the (weak) intrinsic dielectric activity of PS. Again, $\boldsymbol{A}$ (cf. Figure 6b) represents a special case since it possesses two probe-related relaxation processes $\left(\alpha\right.$ and $\left.\beta^{*}\right)$. The corresponding dependencies $\Delta \varepsilon_{\alpha}(\mathrm{c})$ and $\Delta \varepsilon_{\beta *}(\mathrm{c})$ are linear and have nearly 
the same slope, indicating that the probe mean squared dipole moment $\left\langle\mu^{2}\right\rangle$ contributes equally to the two relaxation modes.

Taking advantage of the intrinsic dielectric activity of PS, one has the unique opportunity to compare two different dielectric manifestations of the glass transition dynamics in the same material:

a) A direct one due the presence of intrinsic markers (the "phenyl" dipoles), and

b) an indirect response due to the coupling of probe mobility with the cooperative dynamics of the polymer matrix.

Implications of the dual origin of the dielectric $\alpha$-process will be discussed below.

\section{Insert figure 6.}

\section{Discussion}

\subsection{Minimum probe size for sensing cooperative dynamics}

The peculiar behavior of the shortest probe, both sensing the segmental dynamics of PS and revealing an additional $\beta^{*}$ - process, brings us to the question whether there exist two different molecular reorientation mechanisms that give rise to either an Arrhenius-type (A) or a VFT-type relaxation mode (all probes), and how they are related to the probe length.

Let's first consider a sufficiently long rod-like molecule (length $l$ ), in the sense that its length is much larger than the typical particle size that forms the viscous environment. In this case, the rotational dynamics of the probe in governed by diffusive rearrangements of all particles, here polymer segments, within the hydrodynamic volume $\left(\sim l^{3}\right)$. As a consequence, probe reorientations obey a rotational diffusion mechanism that gives access to all angular positions, i.e. allows full probe relaxation.

The striking feature of the shortest probe $(\boldsymbol{A})$ is its remarkable rotational mobility ( $\beta^{*}$-process) even in the glassy state of the polymer matrix, where environmental fluctuations on the length scale $l$ are frozen in. Instead of rotational diffusion, which is unlikely to occur below $T_{\mathrm{g}}$ as proven by the absence of the $\alpha$-process, rotational jumps with large jump angles [23] are favored to rationalize the dielectric $\beta^{*}$-process. This idea is corroborated by two observations.

i) In a rotational jump scenario, assuming a rigid molecule being captured in an odd-shaped "free-volume" cavity, not all angular positions are accessible. Consequently, the dielectric relaxation strength $\Delta \varepsilon_{\beta^{*}}$ does not represent the total dielectric activity of the probe $\boldsymbol{A}$, as confirmed by the additional probe contribution to the $\alpha$-process (cf. discussion in previous section). 
ii) The abrupt change in the activation energy of the $\beta^{*}$-process around $T_{\mathrm{g}}$ indicates that $E_{\mathrm{a}}$ is strongly related to the temperature dependence of the (free) volume.

Within the concept of truly thermally activated processes, the kink in the $\log \tau_{\beta *}(1 / T)$ dependence is counterintuitive, because it implies that the energy barrier $E_{\mathrm{a}}$ would increase with increasing temperature. Nonetheless, similar phenomena has repeatedly been reported and controversially discussed in the literature for various secondary relaxations in amorphous polymers like Poly(methyl methacrylate) [17, 24].

The easiest way to rationalize this apparent increase in the activation energy is the assumption that the actual relaxation rate also depends on the free volume and its temperature dependence, which typically changes by a factor of 5 upon passing the glass transition. Since an increase of the free volume results in an increase of the relaxation rate, it is clear that thermal expansion of the local free volume will result in a corresponding increase of the apparent, measured activation energy. A more detailed discussion involving recent free volume data from positron annihilation lifetime spectroscopy (PALS) on PS networks [25] will be subject of a forthcoming paper.

The obvious sensitivity of probe $\boldsymbol{A}$ to the local density, by virtue of a rotational jump mechanism, has two implications. First, the $\beta^{*}$-relaxation might be used as a very sensitive and local means to measure the volumetric glass transition. On the other hand, the exclusive observation of the $\beta^{*}$-process for the shortest probe gives a hint about the size of free volume cavities, the effective diameter of which should range between $0.6 \mathrm{~nm}$ and $1.3 \mathrm{~nm}$. These values are in good agreement with results based on photochromic and fluorescent probe studies on PS [26], which suggested a typical size of free volume regions between 0.130 and $0.280 \mathrm{~nm}^{3}$, corresponding to an effective diameter between 0.63 and 0.81 $\mathrm{nm}$.

\subsection{Effects of the probe length on the probe relaxation time}

The initial motivation for using dielectric probes of different length was to probe the cooperative dynamics over an adjustable length scale that should be closely related to the probe length $l$. For the ideal case of a rotational probe that fluctuates in a homogeneous liquid with a given microviscosity, the rotational relaxation time will only depend on the probe length and the viscosity.

Unfortunately, there is compelling evidence that the dynamics of glass forming liquids is rather heterogeneous, i.e. there exist temporary regions where molecules (or polymer segments) possess a faster and a slower dynamics than the average. The implications and the experimental verification of such a heterogeneous scenario have been debated quite extensively in the past years [27, 28]. For simplicity we will now assume that there exist a temperature dependent, characteristic correlation length $\xi(T)$ that describes both the typical size of and the distance between dynamically correlated 
subensembles of cooperatively rearranging "monomers" (the "CRRs" [29]). We then can consider two cases:

i) The characteristic length $\xi$ is far larger than the probe length, $\xi>l$. Here the probe will sense only the dynamics within a single CRR, a situation that might occur very close to $T_{\mathrm{g}}$.

ii) At high frequencies, where $\xi<l$ is a realistic assumption for a probe with $l>1 \mathrm{~nm}$, the probe rotational diffusion will average over the mobility of several CRRs, between which no dynamic correlation exists. In this scenario, which is expected for high temperatures and thus short relaxation times, the probe molecule senses the average mobility of the polymer segments, i.e. the microviscosity on the nm scale.

It should be noted that for both low and high frequencies, the macroscopic dielectric response will represent an average over the heterogeneous dynamics, however averaging takes place on different levels. For $\xi<<l$, each individual probe will probe several CRRs, which gives rise to a time-averaged (broadened) individual probe response that includes both faster and slower spatial regions. In contrast, at low frequencies $(\xi>l)$, individual probe molecules likely sense the mobility of their local environment, which could give rise to a narrower individual probe response. Here, the measured dielectric response will show broadening due to the ensemble average over individual probe responses. Assuming a quasi-homogeneneous dynamics for high frequencies we can relate the probe relaxation time (cf. Figure 7) to predictions for the rotational diffusion time as function of the probe length. According to Broersma [30], the rotational diffusion time for Brownian rigid rods, in the dilute regime, obeys the relation $\tau \propto l^{3}$. Consequently, the relaxation time of the probe-related $\alpha$-process is expected to slow-down progressively with increasing probe length.

This trend is clearly seen in Figure 7 showing the relaxation time dependencies $\tau_{\alpha}(T)$ for all probes in PS. At high temperatures, a fairly parallel shift of the $\tau_{\alpha}(T)$ curves towards longer relaxation times from $\boldsymbol{A} \rightarrow \boldsymbol{B} \rightarrow \boldsymbol{C}$ is manifested. To proof the validity of the $\tau \propto l^{3}$ relation we have evaluated $\tau / l^{3}$ for relaxation time data at $180^{\circ} \mathrm{C}$. The results are given in Table 2 and show a pretty universal value for $\tau / l^{3}$ around 30ns $/ \mathrm{nm}^{3}$, which is very satisfying in view of uncertainties regarding the actual (dynamic) probe lengths and errors in $\tau_{\alpha}$ for $\boldsymbol{A}$ arising from the VFT- extrapolation.

Table 3. Dielectric relaxation time $\tau_{\alpha}$ at $180^{\circ} \mathrm{C}$, probe length and reduced probe length for all dielectric probe types.

\begin{tabular}{ccccc}
\hline probe & $\tau[\mathrm{ns}]$ & $l[\mathrm{~nm}]$ & $l^{3}\left[\mathrm{~nm}^{3}\right]$ & $\tau / l^{3}$ \\
\hline $\mathbf{A}$ & 246 & 1.96 & 7.50 & 32 \\
$\mathbf{B}$ & 44.8 & 1.28 & 2.10 & 21 \\
$\mathbf{C}$ & 11.7 & 0.64 & 0.26 & 45 \\
\hline
\end{tabular}


While the probe length manifests in the expected manner for short relaxation times, no such simple trend is found for the slow dynamics expressed by the glass transition temperature as function of the probe type (cf. Fig. 7b). Restricting ourselves first to the largest probes $\boldsymbol{B}$ and $\boldsymbol{C}$, the longer relaxation times are again found for the longer probe, which can be rationalised by the idea, that a larger probe senses larger subensembles of monomers within a CRR that have a corresponding longer relaxation time.

This argument obviously does not to hold for probe $\boldsymbol{A}$ that (apparently) senses a slower cooperative dynamics (higher $T_{\mathrm{g}}$ ) than the longer probe $\boldsymbol{B}$. A tentative explanation for this odd behaviour is based on the occurrence of the specific $\beta^{*}$ process: While the $\alpha$-process, associated to the rotational dynamics of the large probes $\boldsymbol{B}$ and $\boldsymbol{C}$, likely represents the whole spectrum of orientational mobility within the hydrodynamic probe volume, the orientational degrees of freedoms of $\boldsymbol{A}$ are activated by two separate relaxation modes, $\alpha$ and $\beta^{*}$. Since the $\beta^{*}$-relaxation obviously represents the fast components of the rotational dynamics, these fast modes are missing in the $\alpha$-process, which leads to an apparent shift of the $\alpha$-relaxation spectrum for longer relaxation times.

\subsection{Effects of probe concentration on the $\alpha$-process}

In a previous paragraph, the significant effect of the probe concentration on both the calorimetric (cf. Table 1) and "dielectric" (cf. Table 2) glass transition temperature was revealed and attributed to plasticization of the polymer matrix by the aliphatic tail fraction of the probe molecules. The effect of probe induced $T_{\mathrm{g}}$-reduction is also seen in Fig. 8a that displays the calorimetric glass transition temperatures vs. probe concentration for all three probe types. Fig. 8 a clearly proves both the linearity of the plasticizing effect with respect to the probe concentration, and the validity of $T_{\mathrm{g}}(c) \rightarrow T_{\mathrm{g}}(\mathrm{PS})$ towards zero probe concentration within the error limits.

\section{Insert figure 8.}

The longest probe $\boldsymbol{C}$ behaves differently compared to the shorter versions $\boldsymbol{A}$ and $\boldsymbol{B}$. On the one hand, the maximum $T_{\mathrm{g}}$-reduction at $c=1 \mathrm{wt} \%$ is negligible implying that there is no effective plasticization at all, probably due to the counterbalance between plasticization by the flexible tail and antiplasticization caused by very long aromatic core. On the other hand, when focusing on the samples with the highest probe concentration, there seems to be a concentration effect that is also confirmed by a significant $T_{\mathrm{g}}$-reduction in the "dielectric" $T_{\mathrm{g}}$ (cf. Fig. 8b) when increasing the concentration of $\boldsymbol{C}$ from 0.5 to $1.0 \%$. 
To rationalize the deviating behavior of $\boldsymbol{C}$, we want to recall the physical basis for plasticization. The linearity between plasticiser concentration and the deviation of the glass transition temperature $\Delta T$ from the $T_{\mathrm{g}}$ of the pure polymer matrix relies on the idea, that the addition of plasticiser molecules results in a homogeneous speed-up in the cooperative (glass transition) dynamics. For high concentrations, such assumption is certainly valid provided association effects between plasticiser molecules can be ignored. In the opposite case, a highly diluted concentration of plasticiser molecules, is likely that locally plasticised regions coexist with "unplasticised" regions, which necessarily results in a heterogeneous scenario. Consequently, both the concentration of locally plasticised regions (= concentration of plasticiser molecules) and the range of the plasticising effect determine whether there is sufficient or incomplete overlap between the plasticised regions that gives rise to either the homogeneous or heterogeneous scenario.

Considering local plasticisation as an exclusively dynamic phenomenon, one is temped to identify the intrinsic range of local plasticisation with the length scale of cooperativity $\xi$, i.e. the size of a locally plasticised region corresponds to the typical size of a CRR according to the picture of Adams and Gibbs [29]. Experimental verification of the transition from the homogeneous to the heterogeneous $c^{*}$ would thus allow to determine $\xi$, provided some assumptions about the shape of the CRR and the spatial distribution of the plasticiser molecules can be made.

To get a clue about typical length scales we have computed the probe-probe distance for the various probe types as a function of probe concentration for the simplest case that all probe molecules are arranged on a cubic lattice of the edge length $l$. The results (cf. Fig. 9) show that for our experiments typical probe-probe distances between 3.5 and $9.5 \mathrm{~nm}$ are expected, a range that corresponds well with values for $\xi$ discussed in the literature [31].

\section{Insert figure 9.}

The question is whether we have any experimental evidence for the existence of a critical probe concentration, i.e. for a heterogeneous scenario. Such scenario would be characterized by the existence of two separated glass transition peaks that relate to the unplasticised and the plasticised polymer fraction. In other words, the observation of a double glass transition process would be a direct proof of the heterogeneous scenario. Upon further decrease of the plasticiser concentration, these processes are expected to change their intensity according to the fraction of plasticised/unplasticised material, however, no shift in the individual glass transitions is expected.

Having sketched the signature of the heterogeneous scenario, we want to return to our experimental results. Unfortunately, neither the DSC nor the dielectric relaxation data provide clear evidence for a double glass transition, which is not surprising since the two individual $T_{\mathrm{g}}$ 's are likely separated by only $1-2 \mathrm{~K}$. Such a small difference cannot be directly resolved by DSC measurement and will result 
in an "effective", concentration dependent single $\mathrm{T}_{\mathrm{g}}$ as discussed above. In contrast to the calorimetric response, representing both plasticised and unplasticised regions according to their volume fraction, the probe related dielectric response has the unique advantage to probe selectively the plasticised fraction. Here, the detection of leveling-off in the $T_{\mathrm{g}}(\mathrm{c})$ dependence for $\mathrm{c}<\mathrm{c}^{*}$ would be the equivalent signature of the heterogeneous scenario. It is clear that Fig. $8 \mathrm{~b}$ does not show such a leveling-off, but one has to realize that only for higher probe concentrations $(\geq 0.5 \mathrm{wt} \%)$ the measured dielectric $\alpha$ process is dominated by the probe contribution (cf. Fig. 6a). Taking this $0.5 \%$ as an upper bound for the critical concentration $\mathrm{c}^{*}$, we can derive a lower bound for the critical probe-probe distance from Fig. 9 in the order of $4.5-5.5 \mathrm{~nm}$, which might represent a realistic value for the cooperativity length $\xi$ despite the simplified assumption made in this approach.

Replacing PS by a less polar polymer matrix will allow to explore even lower concentration ranges and should result in a more precise determination of $\mathrm{c}^{*}$.

\section{Conclusions}

The effect of the molecule size and concentration of three dielectric probes on their relaxation properties in a polystyrene matrix was studied systematically. For all probes, representing a variation in the rigid core length from 0.6 to $2.0 \mathrm{~nm}$, a significant enhancement of the intrinsic $\square$ a-relaxation was found that was linear within the whole probe concentration range from $0.1-1.0 \mathrm{wt} \%$.

Apart from the fact that all different probes are able to sense the cooperative glass transition dynamics, various manifestations of their characteristic size were found:

1) The high-temperature probe-related relaxation time was found to scale with the (rigid) probe length according to $\tau \propto l^{3}$, which confirmed rotational diffusion as the linking mechanism between the polymeric segmental dynamics and the probe response.

2) The occurrence of an exclusive, additional Arrhenius-type ( $\left.\beta^{*}\right)$ relaxation for the shortest probe $\boldsymbol{A}$, indicates the existence of a critical lower probe size $\left(l^{*} \sim 0.6 \mathrm{~nm}\right)$ below which large angular probe fluctuations are also enabled via a discrete jump mechanism. This feature qualifies the shortest probe also as a free-volume probe as indicated by the pronounced anormality in the $\tau_{\mathrm{b}} *(T)$ dependence.

Finally, a first attempt was made to relate probe concentration effects on the glass transition temperature (plasticizing effect) to the intrinsic length scale of cooperative dynamics $\xi$. Based on a simple model for local plasticisation and the observation of a linear $T_{\mathrm{g}}(c)$ dependence we have determined a lower bound for $\xi$ being in the order of $5 \mathrm{~nm}$. The use of stronger "plasticising" dielectric probes in combination with a less polar matrix is considered as a promising approach for the experimental determination of $\xi$. 


\section{Acknowledgements:}

Piet Droppert (DRS measurements) and Ben Norder (DSC experiments) are gratefully acknowledged for assisting with the experiments. 


\section{References}

[1] F. Kremer, A. Schönhals, Broadband Dielectric Spectroscopy, Springer-Verlag, Berlin Heidelberg New-York, 2003.

[2] J.P. Runt, J.J. Fitzgerald, Dielectric Spectroscopy of Polymeric Materials: Fundamentals and Applications, Washington, 1997.

[3] C.J.F. Bottcher, P. Bordewijk, Theory of Electric Polarization, Elsevier Scientific Pub. Co., New York, 1978.

[4] N.E. Hill, W.E. Vaughan, A.H. Price, M. Davies, Dielectric Properties and Molecular Behaviour, Van Nostrand Reinhold, London, 1969.

[5] M. Wübbenhorst, A.L. de Rooij, J. van Turnhout, J. Tacx, V. Mathot, Coll. Polym. Sci. 279 (2001) 525-531.

[6] N.G. McCrum, B.E. Read, G. Williams, Anelastic and Dielectric Effects in Polymeric Solids, Dover Publications, New York, 1991.

[7] A. Boersma, J. van Turnhout, M. Wübbenhorst, Macromolecules 31 (1998) 7453-7460.

[8] G.P. Simon, J.P. Runt, W.D.U. ACS, Vol 1, 329 - 378, 1997., in Dielectric Spectroscopy of Polymeric Materials: Fundamentals and Applications, Vol. 1 (Ed.: J. J. Fitzgerald), ACS Books (ACS Professional Reference Series), Washington, 1997.

[9] M. Wübbenhorst, B.J.B. Folmer, J. van Turnhout, R.P. Sijbesma, E.W. Meijer, IEEE Transactions on Dielectrics and Electrical Insulation 8 (2001) 365-372.

[10] C.R. Ashcraft, B. R.H., J. Polym. Sci.: Polym. Phys. Ed. 14 (1976) 2153-2193.

[11] P. Frübing, D. Blischke, R. Gerhard-Multhaupt, M.S. Khalil, Journal of Physics D-Applied Physics 34 (2001) 3051-3057.

[12] O.v.d. Berg, W.G.F. Sengers, W.F. Jager, S.J. Picken, M. Wübbenhorst, Macromolecules 37 (2004) 2460-2470.

[13] The dipole moments of A, B and C were calculated using molecular dynamics software "B3LYP/cc-pV02". For A a dipole moment of $8.1 \mathrm{D}$ was calculated, for B this was $10.8 \mathrm{D}$ and for C $12.1 \mathrm{D}$.

[14] S. Havriliak, S. Negami, Polymer 8 (1967) 161.

[15] K. Levenberg, Quart. Appl. Math 2 (1944) 164.

[16] D. Marquardt, J. Soc. Indust. Appl. Math. 11 (1963)

[17] R. Bergman, F. Alvarez, A. Alegria, J. Colmenero, Journal of Non-Crystalline Solids 235 (1998) 580-583.

[18] There is some debate over this interpretation. In some older reports the $-120^{\circ} \mathrm{C}$ phase transition is interpreted as the glass transition

[19] Y. Jin, R.H. Boyd, Journal of Chemical Physics 108 (1998) 9912-9923.

[20] H.Z. Vogel, Phys. 22 (1921) 645.

[21] G. Tammann, G.Z. Hesse, Anorg. Allg. Chem. 156 (1926) 245.

[22] G.S. Fulcher, J. Am. Ceram. Soc. 8 (1925) 339.

[23] A. Doss, M. Paluch, H. Sillescu, G. Hinze, Journal of Chemical Physics 117 (2002) 65826589.

[24] R. Bergman, F. Alvarez, A. Alegria, J. Colmenero, Journal of Chemical Physics 109 (1998) 7546-7555.

[25] K. Cosaert, F. Du Prez, N. Balcaen, J. De Baerdemaeker, C. Dauwe, D. Cangialosi, M. Wübbenhorst, C. Bas, in IUPAC World Polymer Congres MACRO 2004, Paris, 2004, p. 2 pages.

[26] J.G. Victor, J.M. Torkelson, Macromolecules 21 (1988) 3490-3497.

[27] M.D. Ediger, Annual Review of Physical Chemistry 51 (2000) 99-128.

[28] C.Y. Wang, M.D. Ediger, Journal of Chemical Physics 112 (2000) 6933-6937.

[29] G. Adam, J.H. Gibbs, Journal of Chemical Physics 43 (1965) 139-146.

[30] S. Broersma, Journal of Chemical Physics 32 (1960) 1626-1631.

[31] E. Donth, The Glass Transition. Relaxation Dynamics in Liquids and Disordered Materials, Springer, Berlin Heidelberg, 2001. 


\section{Captions to figures:}

Figure 1. Schematic representation of a dielectric probe.

Figure 2. Chemical structures and size indications of the three dielectric probes.

Figure 3. Dielectric loss $\varepsilon$ ' as a function of temperature and concentration for probes $\boldsymbol{B}$ and $\boldsymbol{C}$ at $f=$ $13 \mathrm{kHz}$.

Figure 4. Dielectric loss $\varepsilon "$ as a function of temperature and probe concentration of $\boldsymbol{A}$ at $13 \mathrm{kHz}$.

Figure 5. Arrhenius diagram for the $\alpha$ and $\beta^{*}$-processes of $\boldsymbol{A}$ doped PS.

Figure 6. a) Relaxation strength $\Delta \varepsilon$ of the dielectric glass transition process vs. probe concentration for PS doped with $\boldsymbol{A}, \boldsymbol{B}$ or $\boldsymbol{C}\left(T=135^{\circ} \mathrm{C}\right)$. The solid lines correspond to linear fits to the $\Delta \varepsilon(\mathrm{c})$ data points. b) Relaxation strength $\Delta \varepsilon$ of the $\alpha$ and $\beta *$-process vs. probe concentration for PS doped with A $\left(T=135^{\circ} \mathrm{C}\right)$.

Figure 7. a) Relaxation time $\log \left(\tau_{\alpha}\right)$ vs. $1 /$ T for PS doped with $0.5 \%$ for probe $\boldsymbol{A}, \boldsymbol{B}$ and $\boldsymbol{C}$. b) $\log \left(\tau_{\alpha}\right)$ at $T=180^{\circ} \mathrm{C}$ as function of the logarithmic probe length. The solid line corresponds to the slope $d \log \left(\tau_{\alpha}\right) / d \log (l)=3$.

Figure 8. a) Calorimetric glass transition temperatures vs. probe concentration according to the DSCdata from Table 1. b) "Dielectric" glass transition temperatures (cf. Table 2) as function of the probe-related aliphatic volume fraction for PS with $\boldsymbol{A}, \boldsymbol{B}$ and $\boldsymbol{C}$.

Figure 9. Minimum probe-probe distances assuming a regular arrangement of probe molecules in a cubic lattice for different probe types and probe (mass) concentration. 


\section{Figure 1:}

Rigid core

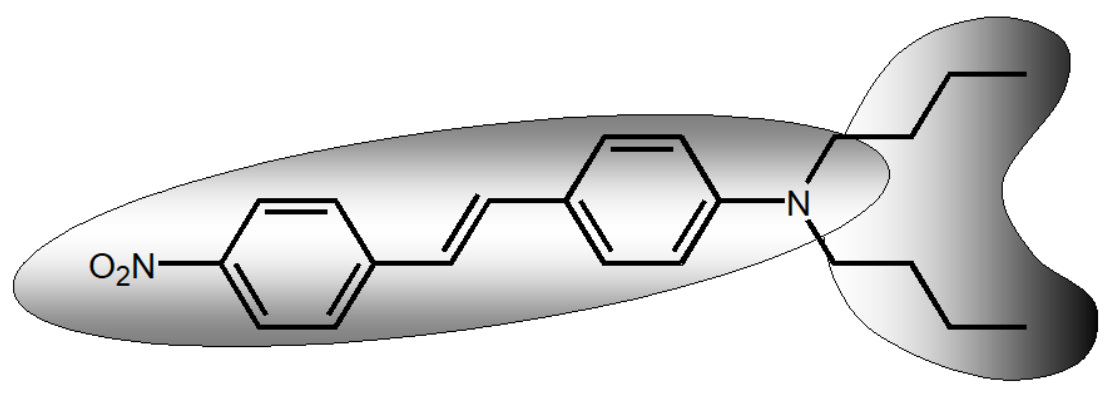


Figure 2.

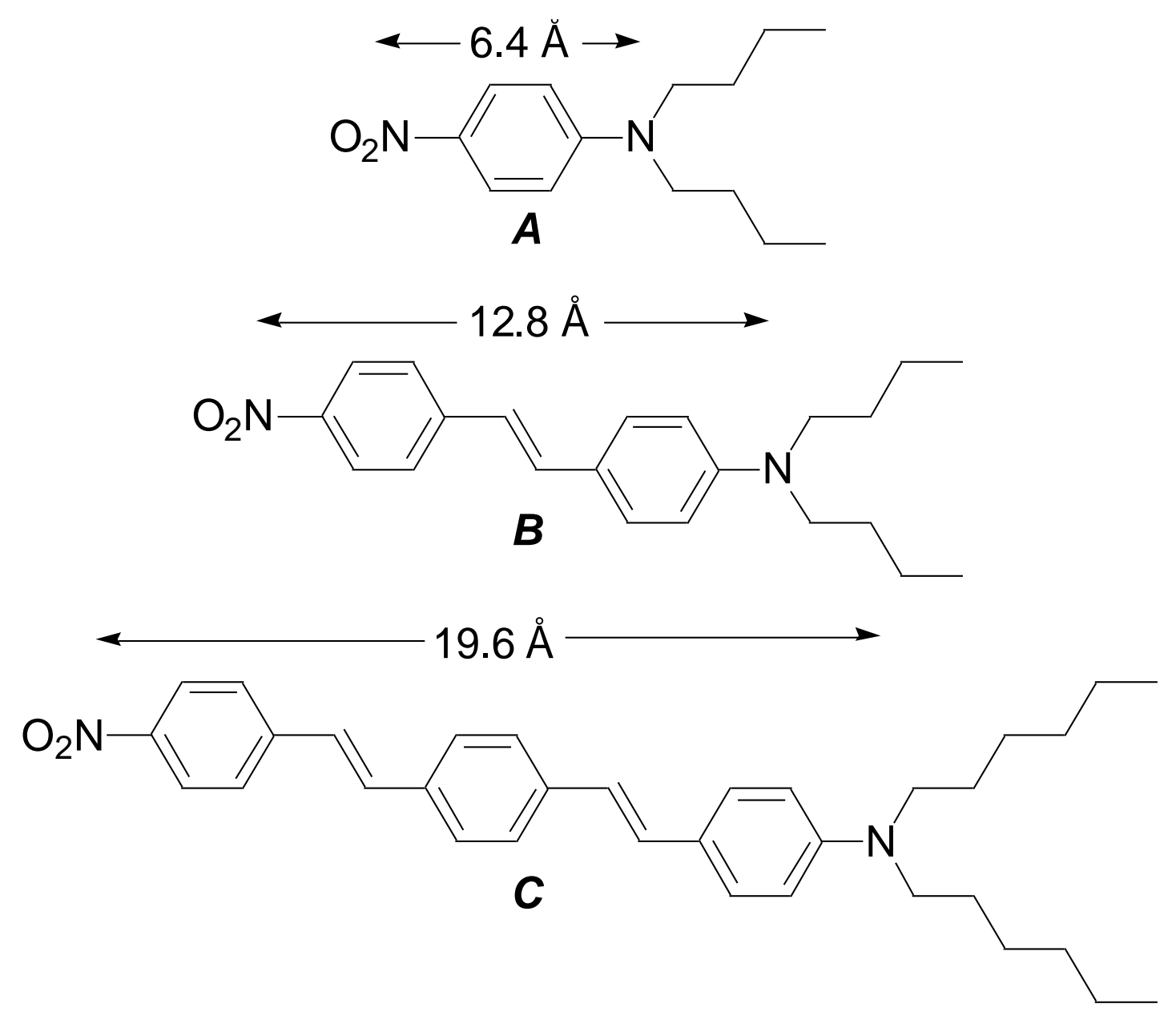


Figure 3.

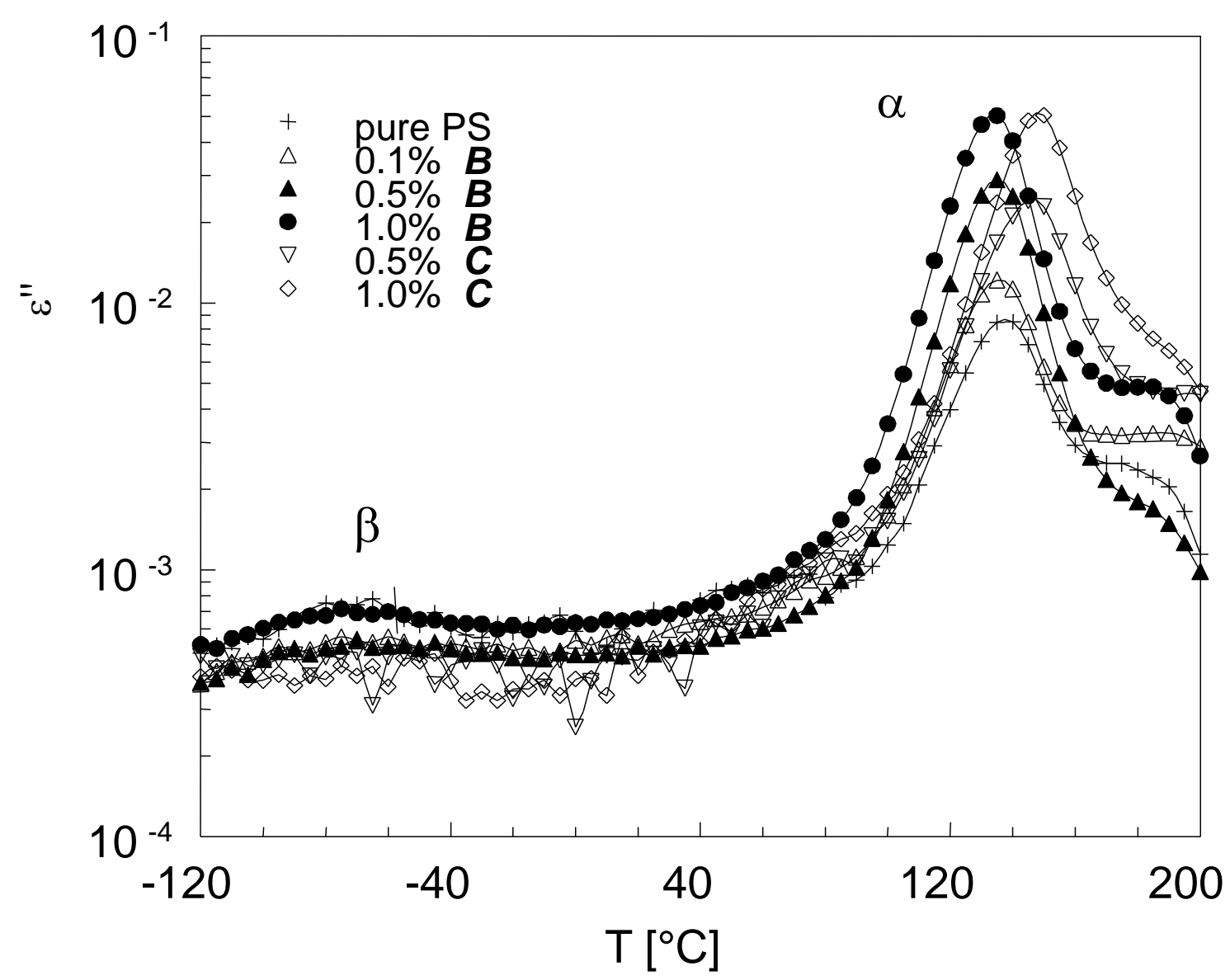


Figure 4.

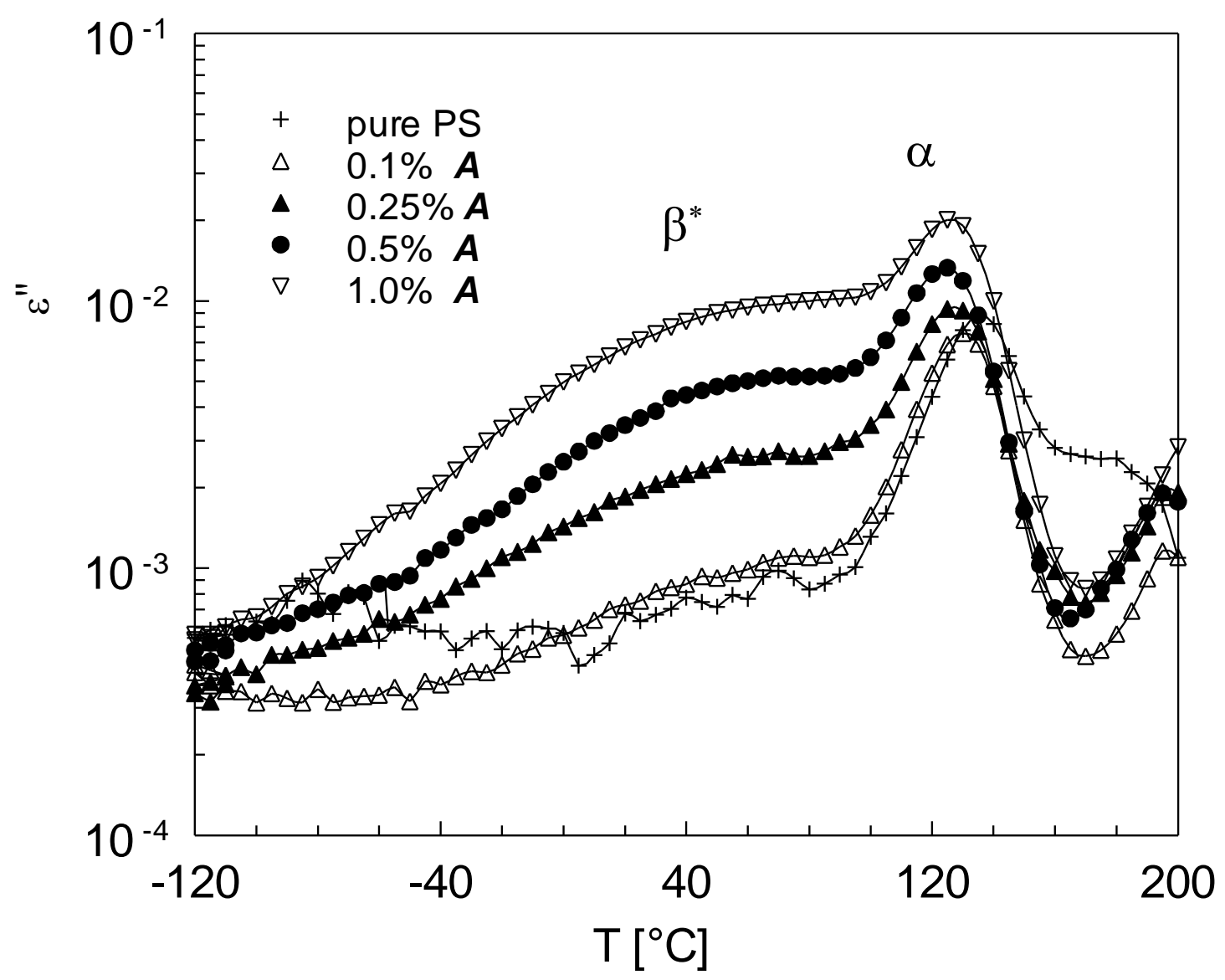


Figure 5.

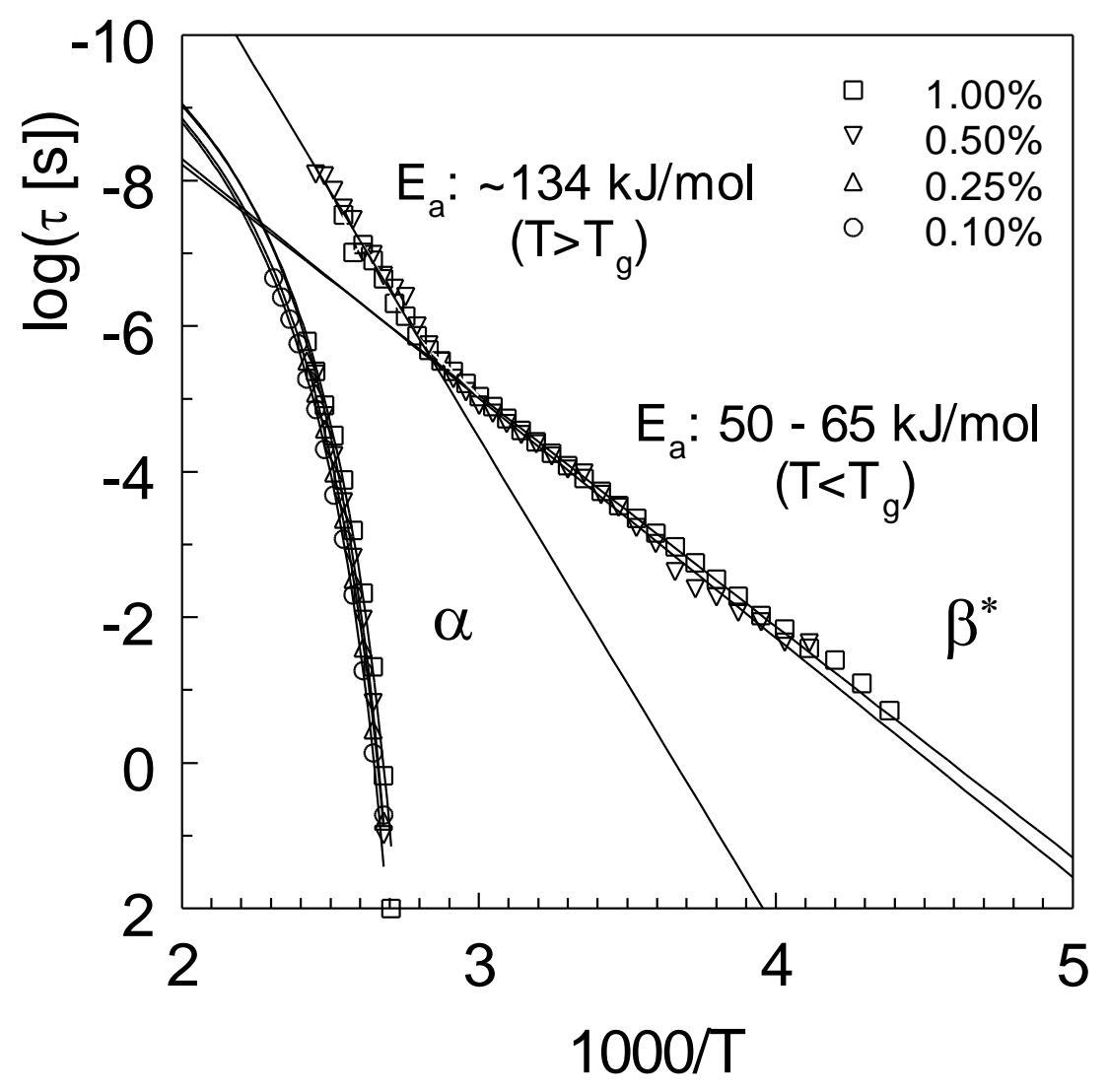


Figure 6.

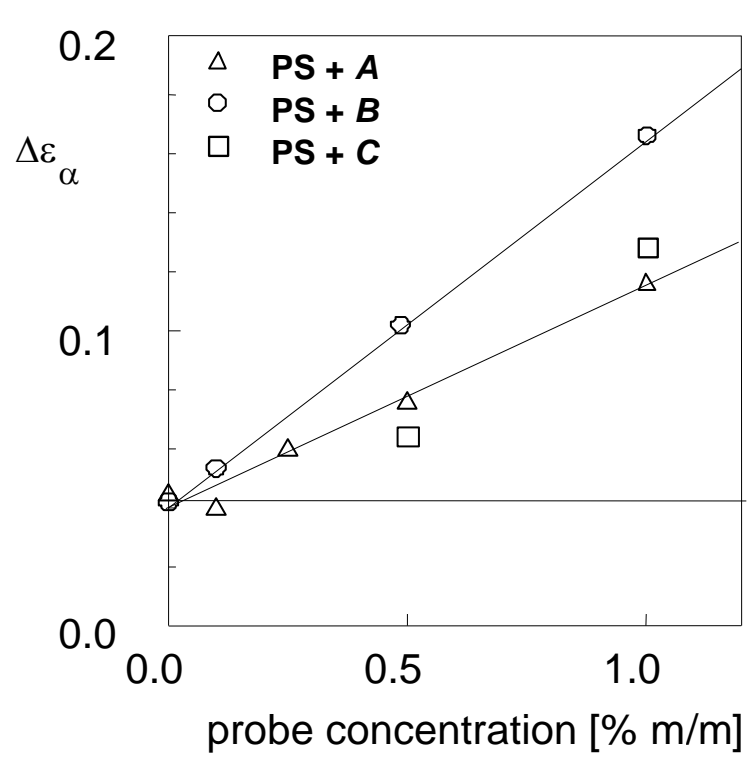

(a)

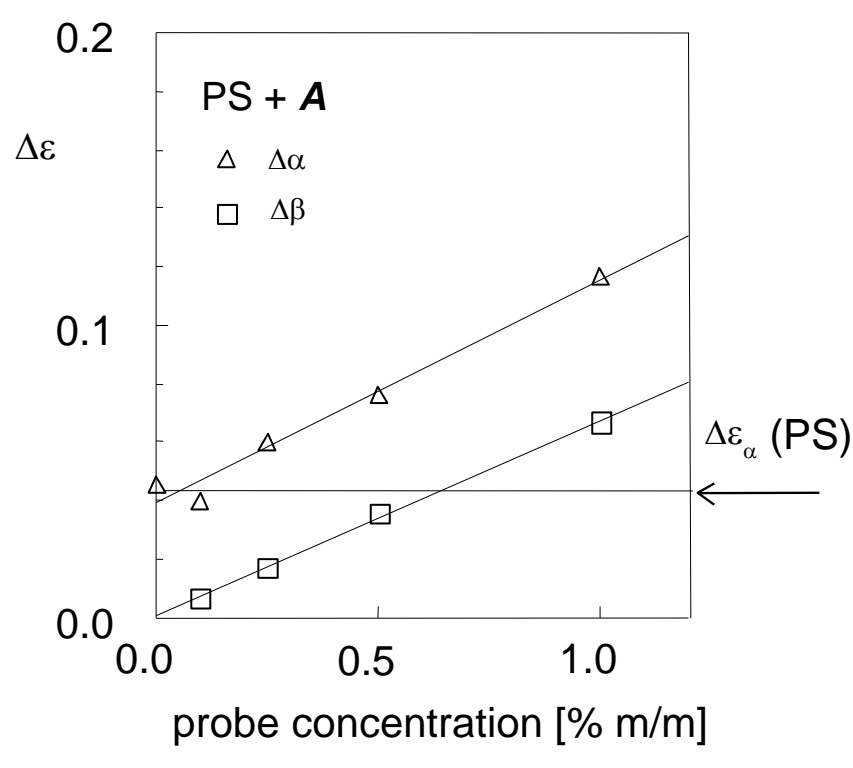

(b) 


\section{Figure 7.}

a)

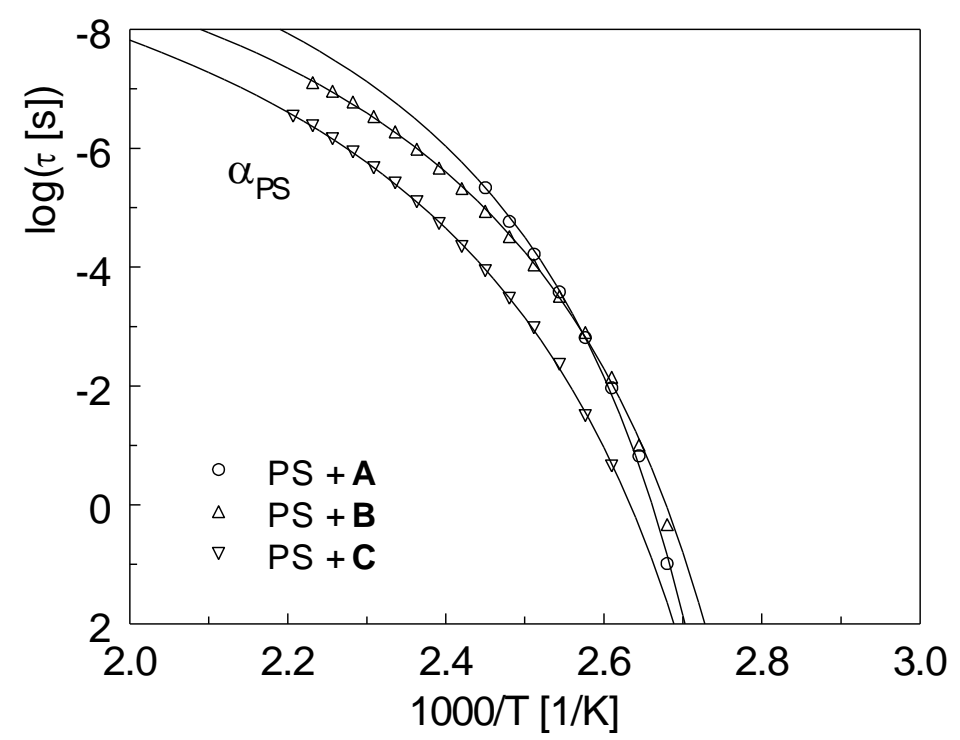

b)

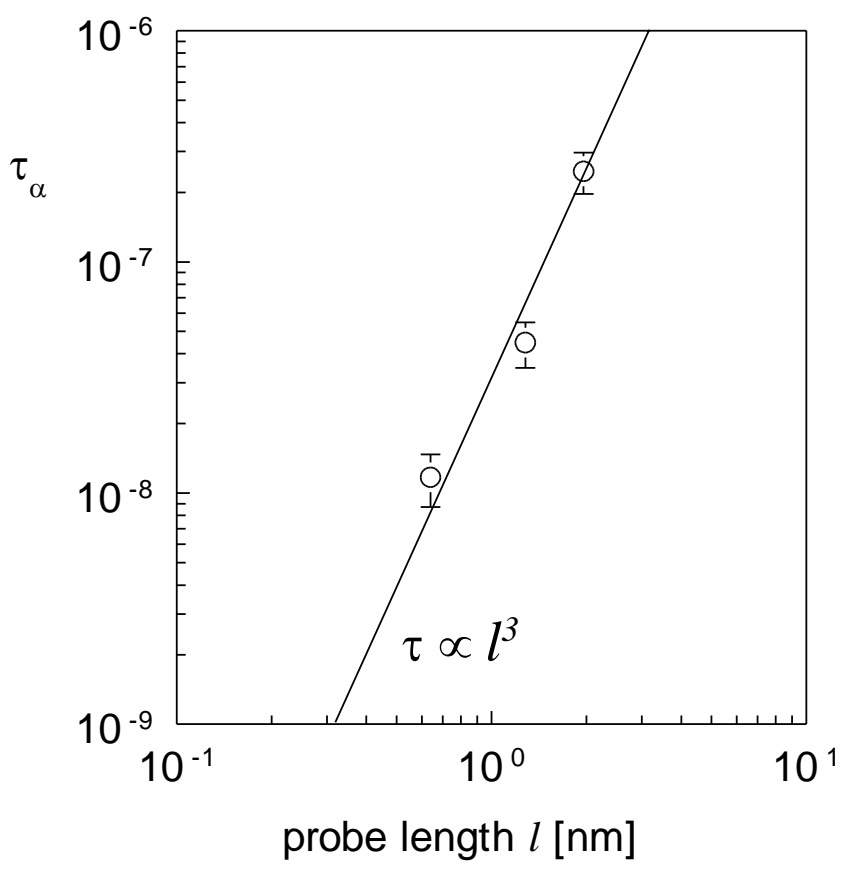




\section{Figure 8.}

a)

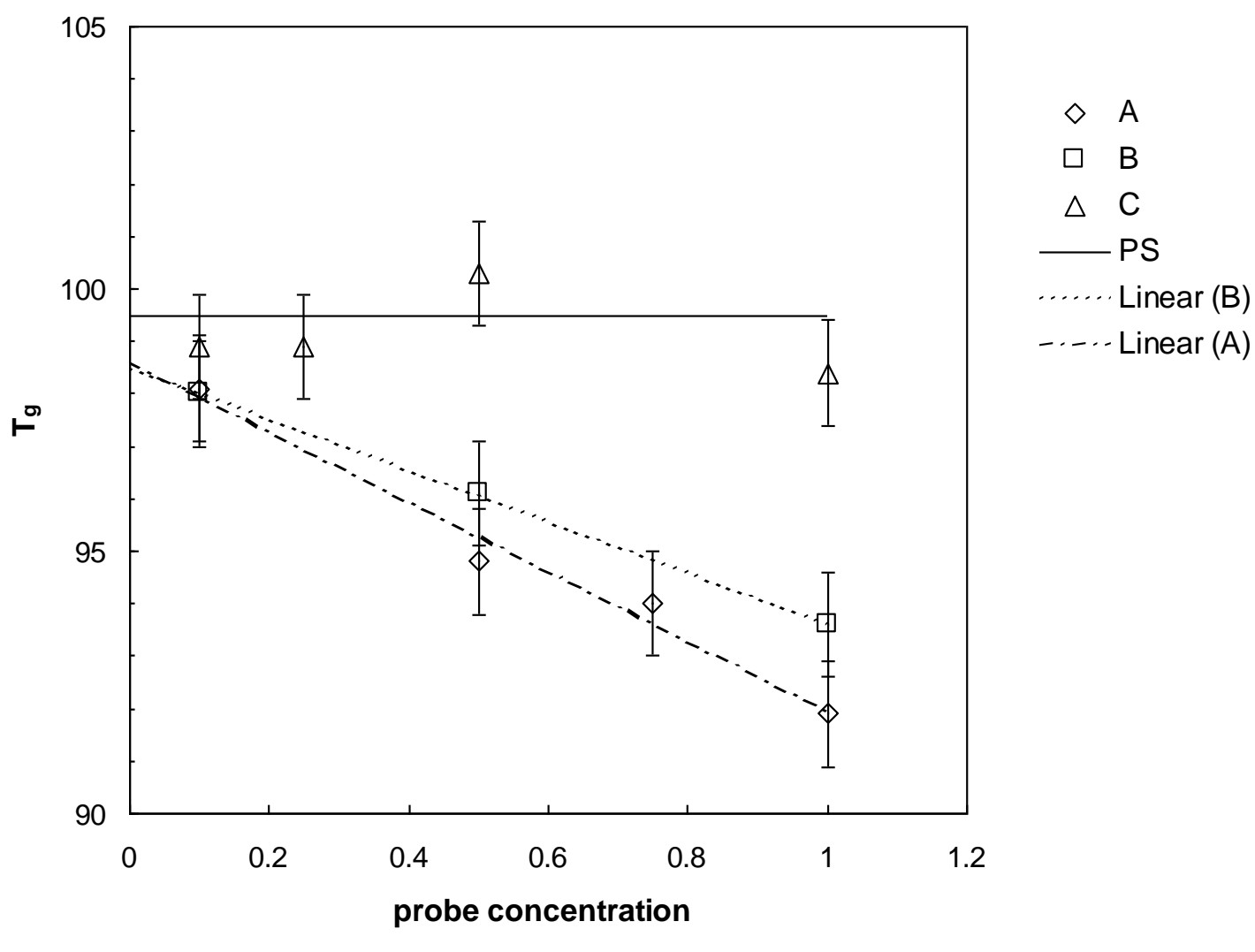

b)

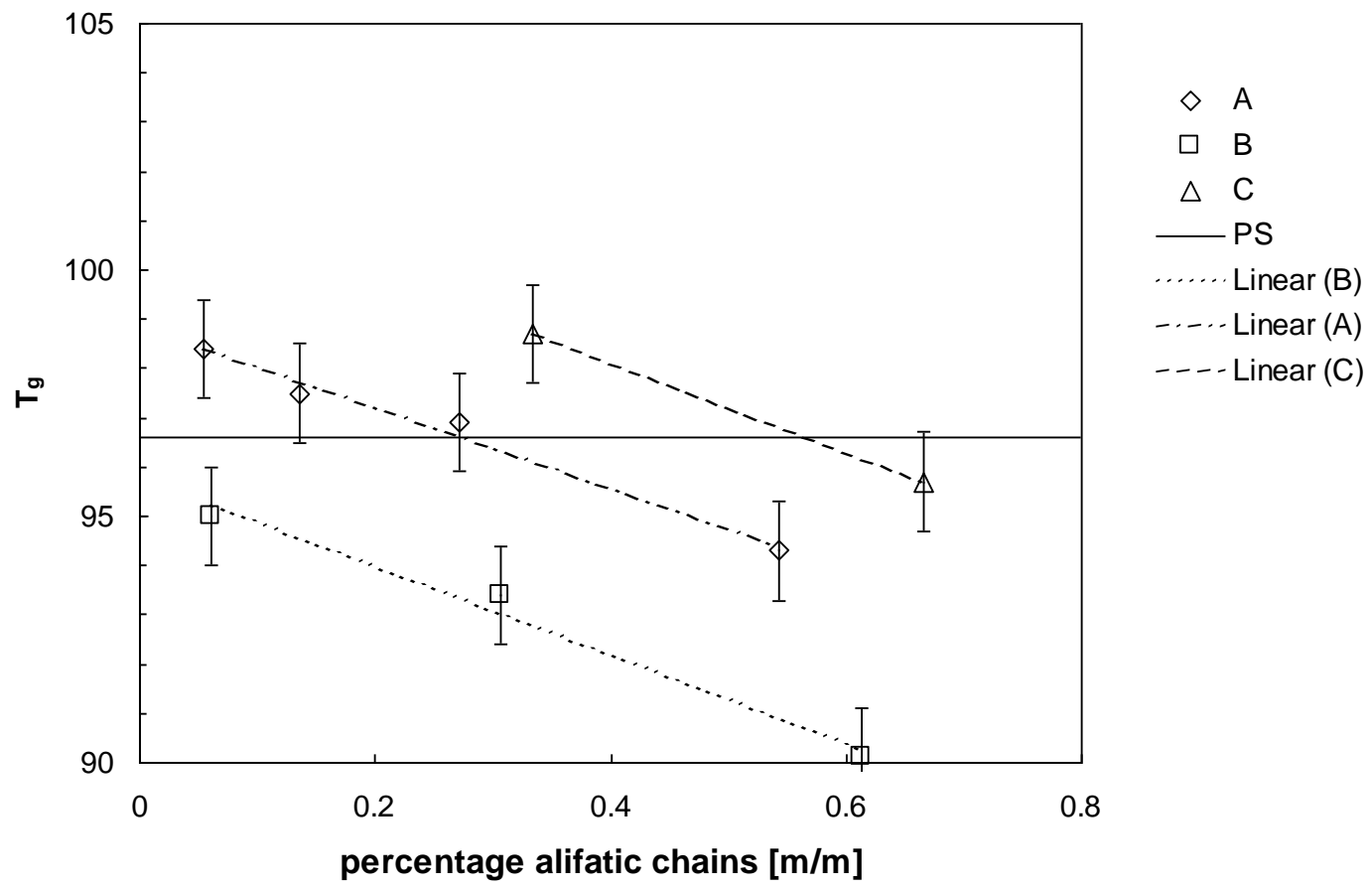


Figure 9.

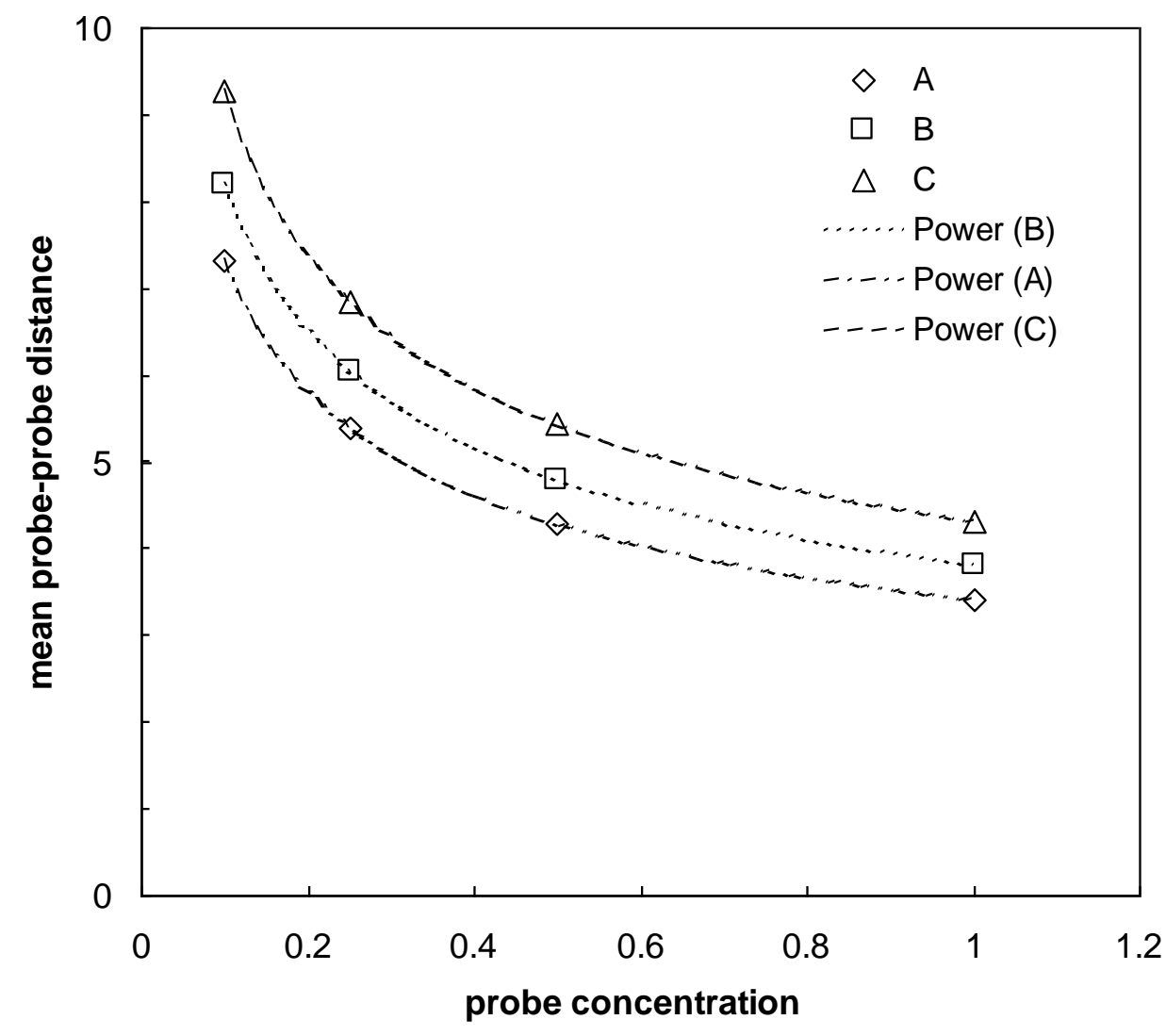

